\title{
Cleavage of Blood Coagulation Factor XIII and Fibrinogen by Thrombin during In Vitro Clotting
}

Charles S. Greenberg, Charles C. Miraglia, Frederick R. Rickles, and Marc A. Shuman

Department of Medicine, Duke University Medical Center, Durham, North Carolina 27710; Department of Medicine and Cancer

Research Institute, University of California, San Francisco, California 94143; and Division of Hematology-Oncology, Department of

Medicine, University of Connecticut Health Center and the Veterans Administration Medical Center, Newington, Connecticut 06111

\begin{abstract}
Thrombin cleavage of blood coagulation Factor XIII $\left(\mathbf{a}_{2} \mathbf{b}_{2}\right)$ and fibrinogen was studied during in vitro clotting to determine the physiologic sequence of these events. First, the time course of fibrin formation and cleavage of Factor XIII was measured in platelet-rich plasma. Cleavage of fibrinogen was measured by using a radioimmunoassay for fibrinopeptide A. Conversion of trace amounts of radioiodinated a-chains of ${ }^{125}$ I-Factor XIII to thrombin-modified a-chains was measured in unreduced $10 \%$ sodium dodecyl sulfate-polyacrylamide gels. During spontaneous clotting, a similar percentage of ${ }^{\mathbf{1 2 5}} \mathrm{I}$-Factor XIII and fibrinogen was cleaved at each time point. Visible gelation of polymerized fibrin monomer occurred when $24 \pm 8 \%$ of fibrinogen was cleaved and $21 \pm 6 \%$ of Factor XIII was converted to Factor XIII'.

Thrombin cleavage of Factor XIII and fibrinogen was also studied in platelet-poor plasma to which thrombin was added. In order to measure Factor XIIIa activity, fibrin polymerization was completely inhibited by the addition of Gly-Pro-Arg-Pro. Factor XIIIa formation was measured by the incorporation of $\left[^{3} \mathrm{H}\right]$ putrescine into casein. The concentration of added thrombin required to cleave $50 \%$ of fibrinogen and Factor XIII was 0.65 $\mathrm{U} / \mathrm{ml}$ and $0.35 \mathrm{U} / \mathrm{ml}$, respectively. The rate of cleavage of fibrinogen by thrombin was 43-fold greater than cleavage of Factor XIII. Lower Gly-Pro-Arg-Pro concentrations were used to determine the effects of incompletely inhibiting fibrin polymerization on cleavage of Factor XIII and fibrinogen. Thrombin cleavage of Factor XIII but not fibrinogen was dependent on the extent of fibrin polymerization. The more marked the degree of inhibition of fibrin polymerization, the slower the rate of Factor XIIIa formation.

Thus, in platelet-rich plasma, thrombin cleavage of Factor XIII and fibrinogen are closely related events during spontaneous clotting. Furthermore, cleavage of Factor XIII during clotting is enhanced by fibrin polymerization in platelet-poor plasma.
\end{abstract}

\section{Introduction}

Formation and stabilization of blood clots require a complex interaction involving conversion of fibrinogen to fibrin and

A preliminary report of this work was presented at the annual meeting of the American Society for Clinical Investigation, May 1982, in Washington, DC, and was published as an abstract (1982. Clin. Res. 30:503A).

Received for publication 3 January 1983 and in revised form 15 January 1985.

J. Clin. Invest.

(c) The American Society for Clinical Investigation, Inc. $0021-9738 / 85 / 05 / 1463 / 08 \quad \$ 1.00$

Volume 75, May 1985, 1463-1470 activation of plasma Factor XIII. The conversion of fibrinogen to a cross-linked gel occurs in several steps. Thrombin cleaves the amino-terminal regions of the $A \alpha$ and $B \beta$ chains of fibrinogen and releases 2 mol each of fibrinopeptide A (FPA) ${ }^{1}$ and fibrinopeptide B (FPB) for each mole of fibrin monomer produced $(1,2)$. The release of FPA has been shown to be more rapid than that of FPB (2). The fibrin monomers first spontaneously polymerize end-to-end to form protofibrils, which then associate laterally to form fibrin fibers $(2,3)$. These fibrin fibers form a network and the final fibrin solution is converted to a gel when $\sim 25 \%$ of the fibrinogen is converted to fibrin (4).

Plasma Factor XIII is converted to the active Factor XIIIa in two steps. In the first step, thrombin cleaves a 4,000-mol wt peptide from each of the two a-chains of plasma Factor XIII (5) with formation of an inactive intermediate, Factor XIII', $\left(a_{2}^{\prime} b_{2}\right)(6)$. Then in the final step, calcium causes the $\mathbf{b}_{2}$-subunit to dissociate forming Factor XIIIa (a $\left.\mathbf{a}_{2}^{*}\right)$ (6). Fibrinogen and fibrin in vitro interact with Factor XIII' to reduce the calcium concentration required to dissociate the b-chains and expose the active site (7). Fibrinogen lowers the concentration of thrombin required for cleavage of plasma Factor $\mathrm{XIII}$ in vitro (8).

When blood clots in vitro, Factor XIIIa activity is reduced in serum compared to plasma (9). Immunologic studies also indicate that the $b$-chains are in serum whereas the thrombincleaved a-chains are bound to fibrin $(10,11)$. Within the fibrin clot, Factor XIIIa first catalyzes formation of $\gamma$-glutamyl $\epsilon$-lysyl bonds between $\gamma$-chains and then cross-links the $\alpha$ chains of fibrin monomers (12).

The time course of fibrin formation and Factor XIII cleavage by thrombin has not been previously studied under physiologic conditioning. In this paper, we report the results of studies of in vitro clotting designed to determine when Factor XIII is cleaved by thrombin relative to fibrin formation, and whether fibrin polymerization modifies thrombin cleavage of Factor XIII.

\section{Methods}

Materials. All chemicals were reagent grade or highest quality available. Carrier-free sodium ${ }^{125}$ I-iodide for protein iodination and $\left[{ }^{3} \mathrm{H}\right]$ putrescine were purchased from Amersham Corp., Arlington Heights, IL. Polystyrene test tubes, $12 \times 75 \mathrm{~mm}$, were obtained from VWR Scientific Div., Univar, San Francisco, CA. Human fibrinogen (grade L) was purchased from Kabi, Inc., Greenwich, CT. Sodium dodecyl sulfatepolyacrylamide gel electrophoresis (SDS-PAGE) molecular weight stan-

1. Abbreviations used in this paper: FPA, fibrinopeptide A; FPB, fibrinopeptide B; PA, polyacrylamide; PAGE, polyacrylamide gel electrophoresis; PNPGB, para-nitrophenylguanidinobenzoate; PPP, plateletpoor plasma; PRP, platelet-rich plasma. 
dards and electrophoresis reagents were obtained from Bio-Rad Laboratories, Richmond, CA. Human IgG was supplied by Cappel Laboratories, West Chester, PA. Sodium heparin from beef lung was obtained from Upjohn Co., Kalamazoo, MI. Soybean trypsin inhibitor was obtained from Worthington Diagnostic Systems, Inc., Freehold, NJ. Gly-Pro-Arg-Pro was purchased from Vega Biotechnologies, Tucson, AZ. Hirudin and para-nitrophenylguanidinobenzoate (PNPGB) were obtained from Sigma Chemical Co., St. Louis, MO. Human $\alpha$ thrombin was a gift of Dr. J. W. Fenton II, New York State Department of Health, Albany, NY.

Protein purification. Plasma Factor XIII was isolated from freshfrozen plasma and Cohn fraction I paste by following published techniques (13). Plasma Factor XIII was stored at $-70^{\circ} \mathrm{C}$ in $50 \mathrm{mM}$ sodium citrate, $150 \mathrm{mM} \mathrm{KCl}, \mathrm{pH} 7.4$ (14).

Human fibrinogen was isolated from fresh-frozen plasma by a previously published method which removes contaminating plasminogen, Factor XIII, and fibronectin (15). Concentration of the plasma Factor XIII and fibrinogen was calculated on the basis of $E 1 \% / 1 \mathrm{~cm}$ $=13.8$ and 15.1 , respectively. The percent clottable fibrinogen was determined by standard methods (16).

Radiolabeling of proteins. $80 \mu \mathrm{g}$ of purified protein was radioiodinated at room temperature by the lactoperoxidase-glucose oxidase method as previously described (15). This method has been shown to preserve $\geq 92 \%$ of the transglutaminase activity of Factor XIIIa (15). The specific radioactivity of the proteins varied from 2,200 to 6,900 $\mathrm{cpm} / \mathrm{ng}$. $>95 \%$ of the radiolabel was precipitated with $10 \%$ trichloroacetic acid in the presence of $1 \%$ bovine serum albumin. There was $\sim 0.25$ $0.75 \mathrm{~mol}$ of ${ }^{125} \mathrm{I}$ per mol of Factor XIII.

Gel electrophoresis. SDS-PAGE in $10 \%$ polyacrylamide slab gels with a 3\% stacking gel was performed according to the method of Laemmli (17). Radioiodinated samples were evaluated after electrophoresis by autoradiography on Kodak X-Omat AR x-ray film (Eastman Kodak Co., Rochester, NY) and counting radioactivity in gel slices.

Discontinuous PAGE at pH 7.8 in polyacrylamide (PA) slab gels was performed by standard methods but by using an $8 \%$ gel rather than a $7.5 \%$ gel (6).

Factor XIIIa assays. Factor XIIIa was assayed in heat- and glyceroltreated plasma and serum samples containing ${ }^{125} \mathrm{I}$-plasma Factor XIII by measuring incorporation of monodansylcadaverine or $\left[{ }^{3} \mathrm{H}\right]$ putrescine into $N, N$ dimethylcasein $(18,19)$. The functional activity of purified plasma Factor XIII was determined by titration of the active site cysteines with iodo[ $\left.\left(1-{ }^{14} \mathrm{C}\right)\right]$ acetamide as described by Curtis et al. (6).

Time course of thrombin cleavage of Factor XIII during in vitro clotting of platelet-rich plasma. Blood was collected by the two-syringe technique from normal volunteers. Freshly obtained blood was used in all experiments. Platelet-rich plasma (PRP) was prepared by centrifugation of unanticoagulated blood at $1,600 \mathrm{~g}$ for $3 \mathrm{~min}$ in new polypropylene centrifuge tubes.

$1 \mu \mathrm{g}$ of ${ }^{125} \mathrm{I}$-Factor XIII (0.5 $\mu \mathrm{g}$ of a-chain) was added to $1.0-\mathrm{ml}$ aliquots of PRP in new polystyrene tubes or $0.9-\mathrm{ml}$ aliquots of plateletpoor plasma (PPP) in new borosilicate tubes. Based on the plasma concentration of Factor XIII a-chain of $15 \mu \mathrm{g} / \mathrm{ml}(210 \mathrm{pmol} / \mathrm{ml})(11)$, the final Factor XIII a-chain contribution was $\sim 260 \mathrm{pmol} / \mathrm{ml}$. The PRP samples were allowed to clot at $37^{\circ} \mathrm{C}$ except for control samples, which were anticoagulated with $3.8 \%$ (wt/vol) sodium citrate diluted 1:10. At various intervals before and after clotting, $0.1 \mathrm{ml}$ of $50 \mathrm{U} / \mathrm{ml}$ heparin, $100 \mathrm{mM} \epsilon$-aminocaproic acid (EACA), $50 \mathrm{mM}$ iodoacetic acid, and $100 \mathrm{mM} \mathrm{EDTA}^{2}$ was added to samples and thrombin cleavage of ${ }^{125} \mathrm{I}$-Factor XIII a-chains to $\mathrm{a}^{\prime}$-chains measured by the following technique. Serum and plasma samples were diluted 1:1 with a mixture of SDS-sample buffer which contained $30 \%$ glycerol, $4 \%$ SDS, $8 \mathrm{M}$ urea, $4 \mathrm{mM}$ EDTA, and $0.2 \mathrm{M}$ Tris-Cl, $\mathrm{pH}$ 7.0. Clots were compressed with wooden applicator sticks to remove excess unbound material and then washed three times for $5 \mathrm{~min}$ each in $5 \mathrm{ml}$ of Tris-

2. This mixture of inhibitors was found to block subsequent thrombin cleavage of Factor XIII and Factor XIIIa cross-linking of fibrin.
EDTA buffer $(0.02 \mathrm{M}$ Tris- $\mathrm{HCl}, 0.13 \mathrm{M}$ sodium chloride, $10 \mathrm{mM}$ EDTA, pH 7.0). The clots were then dissolved by incubation in 0.5 $\mathrm{ml}$ of SDS sample buffer at $56^{\circ} \mathrm{C}$ for $18 \mathrm{~h}$. Solubilized samples were electrophoresed in 10\% SDS-PAGE under nonreducing conditions, and the radioactivity in the a- and/or a'-chain was measured. All samples were assayed in triplicate. The amount of the total plasma Factor XIII a-chain cleaved by thrombin was calculated by the formula: ${ }^{125}$ I-plasma Factor XIII a-chain cleaved $(\%) \times\left[{ }^{125}\right.$ I-plasma Factor XIII a-chain (picomoles per milliliter) + endogenous plasma Factor XIII achain (picomoles per milliliter). Untreated and thrombin-treated ${ }^{125} \mathrm{I}$ Factor XIII were electrophoresed simultaneously and used as markers for the $a-, a^{\prime}-$, and $b$-chains.

Thrombin cleavage of fibrinogen during in vitro clotting of PRP. These experiments were carried out in parallel and at the same time as those in which thrombin cleavage of ${ }^{125}$ I-Factor XIII was studied. Experimental conditions were similar to those described for measurement of Factor XIII cleavage except that ${ }^{125}$ I-Factor XIII was not added to the incubation mixture. Fibrin formation was determined by measuring the release of FPA in duplicate at each time point and reported as nanomoles of FPA released per minute (20). The samples were assayed in triplicate.

Time course of thrombin cleavage of Factor XIII and fibrinogen in PPP containing Gly-Pro-Arg-Pro. PPP was prepared by centrifugation of PRP for $15 \mathrm{~min}$ at $500 \mathrm{~g}$. The plasma was then diluted to a final concentration of $20 \%$ (vol/vol) with $0.02 \mathrm{M}$ Tris-HCl, $0.13 \mathrm{M} \mathrm{NaCl}$, pH 7.4, containing Gly-Pro-Arg-Pro. Thrombin was added and at various intervals, inhibitors of thrombin (heparin, $1 \mathrm{U} / \mathrm{ml}$ and PNPGB $0.1 \mathrm{mM}$ ) were added and samples assayed for FPA and Factor XIIIa. In these experiments, FPA was assayed with a kit produced by Mallinckrodt, Inc., St. Louis, MO. The formation of Factor XIIIa in plasma was assayed by a recently reported technique (21). Samples were diluted to a final plasma concentration of $2.5 \%$ ( $\mathrm{vol} / \mathrm{vol}$ ) in 0.1 $\mathrm{M}$ Tris- $\mathrm{HCl}, \mathrm{pH} 8.5$, containing $25 \%$ glycerol, $5 \mathrm{mM} \mathrm{CaCl}_{2}$, and 0.25 $\mathrm{mM}$ Gly-Pro-Arg-Pro. Then, $0.05 \mathrm{ml}$ of the sample was added to 0.01 $\mathrm{ml}$ of dithiothreitol $(200 \mathrm{mM}), 0.010 \mathrm{ml} \mathrm{CaCl} 2(50 \mathrm{mM}), 0.010 \mathrm{ml}$ of $\left[{ }^{3} \mathrm{H}\right]$ putrescine $(100 \mathrm{mCi} / \mathrm{ml}), 0.01 \mathrm{ml}$ of putrescine $(0.250 \mathrm{mM})$, and $0.01 \mathrm{ml}$ of Hammersten casein $(12.5 \mathrm{mg} / \mathrm{ml})$. The mixture was incubated for $60 \mathrm{~min}$ at $37^{\circ} \mathrm{C}$. The reaction was stopped by adding $0.1 \mathrm{ml}$ of $50 \%$ (vol/vol) trichloroacetic acid. Precipitates were collected on Whatman GF/C filters (Whatman Inc., Clifton, NJ) using a Millipore manifold (Millipore Inc., Bedford, MA), then washed with $0.15 \mathrm{ml}$ of $5 \%(\mathrm{vol} / \mathrm{vol})$ trichloroacetic acid. Filters were transferred to glass vials and $10 \mathrm{ml}$ of Aquasol-2 scintillation fluid (New England Nuclear, Boston, MA) was added. Radioactivity was quantitated using a Packard Tricarb spectrometer (Packard Instrument Co., Downers Grove, IL) at a counting efficiency of $28 \%$ for tritium. The assay is linear with respect to time and concentration of plasma. Factor XIII activity was stable for at least $60 \mathrm{~min}$ in $2.5 \%$ plasma with $25 \%$ glycerol.

Measurement of fibrin polymerization and activation of Factor XIII in plasma containing Gly-Pro-Arg-Pro. PPP was diluted to a final concentration of $20 \%(\mathrm{vol} / \mathrm{vol})$ in buffer containing $0.02 \mathrm{M}$ Tris- $\mathrm{HCl}$,

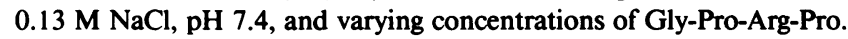
To measure fibrin polymerization, samples were incubated with 0.50 $\mathrm{U} / \mathrm{ml}$ of thrombin at $22^{\circ} \mathrm{C}$ and the change in OD $650 \mathrm{~nm}$ measured in an LKB spectrophotometer (LKP Instruments, Inc., Gaithersburg, MD) with a $10 \mathrm{~mm}$ light path. Factor XIIIa formation was measured in serial aliquots of plasma treated in an identical fashion. Samples $(0.05 \mathrm{ml})$ were mixed with heparin $(0.01 \mathrm{U} / \mathrm{ml})$ and PNPGB $(1.0 \mathrm{mM})$ to inhibit thrombin, then assayed for Factor XIIIa as described in the preceding section.

\section{Results}

Characterization of labeled and unlabeled proteins. The purity of the plasma Factor XIII preparation was analyzed by $10 \%$ SDS-PAGE under reducing and nonreducing conditions. 


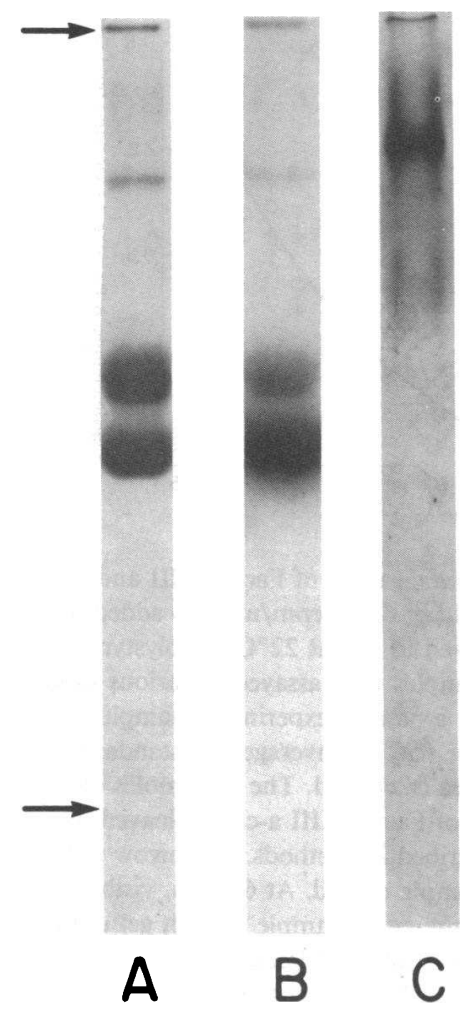

Figure 1. Gel electrophoresis of ${ }^{125} \mathrm{I}$ - and unlabeled plasma Factor XIII. Lane $A$ shows the Coomassie Blue staining pattern of unreduced unlabeled plasma Factor XIII electrophoresed in a $10 \%$ discontinuous SDS-PA gel. Lane $B$ is an autoradiogram of ${ }^{125} \mathrm{I}$-plasma Factor XIII electrophoresed in the same gel as lane $A$ and developed for $14 \mathrm{~h}$. Lane $C$ is the Coomassie Blue staining pattern of plasma Factor XIII electrophoresed in an $\mathbf{8 . 0 \%}$ discontinuous PA gel system as described in Methods. The same amount of protein $(15 \mu \mathrm{g})$ is present in lanes $A$ and $C$. Protein was stained using Coomassie Blue. The top arrow marks the top of the gels. The bottom arrow marks the distance that the bromphenol blue dye marker migrated.

Fig. 1, lane $A$, shows the a-chains and b-chains of unreduced Factor XIII. Densitometric scanning of Coomassie-stained gels demonstrated that $42 \%$ of the protein was in the a-chain area, $46 \%$ in the b-chain, and $12 \%$ in a higher molecular weight band which probably represents undissociated subunits of plasma Factor XIII. When the plasma Factor XIII was reduced and alkylated, there was no band in the high molecular weight region, and the a- and b-chains co-migrated with an $M_{\mathrm{r}}$ $=82,000$ (data not shown). This result is in contrast to previously published studies using a continuous rather than a discontinuous SDS buffer system (4).

When plasma Factor XIII was electrophoresed in a nonSDS discontinuous PA system, a single major band was seen and a smaller amount of another band (Fig. 1, lane $C$ ). The minor band represents free b-chains since purified b-chains migrate in the same region (data not shown).

To determine the extent of radiolabeling of the a- and bchains of plasma Factor XIII, unreduced ${ }^{125}$ I-plasma Factor XIII was subjected to SDS-PAGE (lane $B$ ). Five samples were electrophoresed and the mean \pm standard deviation obtained for the radioactivity in gel slices corresponding to the a-chain subunit was $22 \pm 3 \%$ and $72 \% \pm 4 \%$ in the b-chain subunit; the remainder was in the high molecular weight complex.
Titration of the Factor XIII preparation with $\left[{ }^{14} \mathrm{C}\right]$ iodoacetamide after thrombin treatment in the presence of $10 \mathrm{mM}$ calcium chloride indicated that $63 \%$ of the active site sulfhydryl groups were alkylated. When ${ }^{125} \mathrm{I}$-Factor XIII was activated with thrombin, it retained $87 \%$ of its transglutaminase activity.

Method for measurement of cleavage and activation of ${ }^{125} I$ Factor XIII by thrombin. To study the time course of activation of Factor XIII in plasma, it was necessary to develop sensitive techniques for detecting thrombin cleavage of Factor XIII and to demonstrate that cleavage correlated with formation of Factor XIIIa. ${ }^{125} \mathrm{I}$-Factor XIII and $1 \mathrm{U} / \mathrm{ml}$ of thrombin were incubated at room temperature in $0.02 \mathrm{M}$ Tris- $\mathrm{HCl}, 0.13 \mathrm{M}$

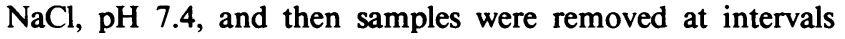
and placed either in SDS buffer or assayed for Factor XIIIa. The samples in SDS were electrophoresed, and conversion of plasma Factor XIII a-chains $(82,000 \mathrm{~mol} w \mathrm{w})$ to $\mathrm{a}^{\prime}$-chain $(78,000 \mathrm{~mol} \mathrm{wt})$ measured. As shown in Fig. 2, after $30 \mathrm{~min}$ of incubation, $92 \% \pm 11 \%$ (mean for three samples \pm standard error) of the radioactivity originally in the a-chain area migrated in the $\mathrm{a}^{\prime}$-chain area. $<5 \%$ of the a-chains of ${ }^{125} \mathrm{I}$-Factor XIII migrated with the $\mathrm{a}^{\prime}$-chains indicating minimal activation prior to treatment with thrombin.

Factor XIIIa formation, assayed in samples treated with heparin and PNPGB to inhibit thrombin, correlated with cleavage of ${ }^{125} \mathrm{I}$-Factor XIII (Fig. 2). When labeled and unlabeled plasma Factor XIII were assayed for transglutaminase activity prior to activation, $<10 \%$ of the activity present after thrombin treatment was detected.

We next demonstrated that ${ }^{125}$ I-Factor XIII and native plasma Factor XIII were cleaved similarly by thrombin. As shown in Fig. 3, appearance of endogenous Factor XIIIa

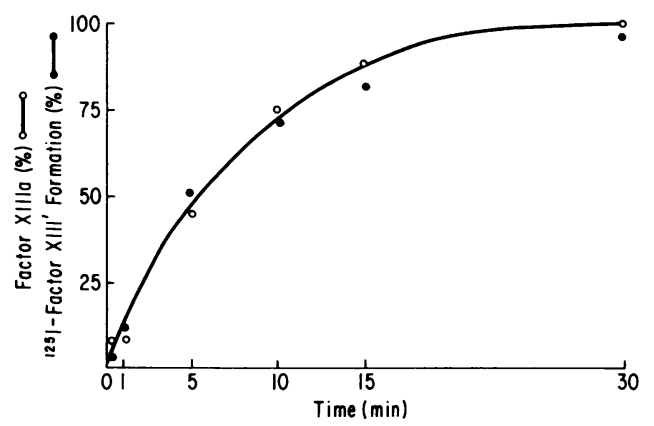

Figure 2. Activation of ${ }^{125} \mathrm{I}$-plasma Factor XIII by thrombin. ${ }^{125} \mathrm{I}$ plasma Factor XIII $(2.5 \mu \mathrm{g} ; 3,250 \mathrm{cpm} / \mathrm{ng})$ was incubated with $1.0 \mathrm{U}$ of thrombin in $1.0 \mathrm{ml}$ of TBS at $22^{\circ} \mathrm{C}$. At various intervals, $0.05 \mathrm{ml}$ of the reaction mixture was removed and subjected to electrophoresis or assayed for Factor XIIIa. Samples were electrophoresed under nonreducing conditions in 10\% SDS-PA gels. Autoradiography of gels was used to identify the a- and a'-chains. Autoradiograms were developed for $24 \mathrm{~h}$ to enhance detection of components of Factor XIII not cleaved by thrombin. The radioactivity in gel slices corresponding to the a- and a'-chains was measured in a gamma spectrometer. The percentage of Factor XIII' formed at various times was calculated by determining the ratio of radioactivity in the $a^{2}$-chain divided by the total amount of radioactivity in the a-chain and $\mathrm{a}^{\prime}$-chain area multiplied by 100 . Each time point $(\bullet)$ represents the mean of three determinations. The standard deviation from the mean was $<8 \%$ for all points measured. Aliquots removed for assay of Factor XIIIa were immediately treated with heparin $(10.0 \mathrm{U} / \mathrm{ml})$ and PNPGB (1.0 mM) to inactivate thrombin. Each time point (o) was assayed in duplicate. 


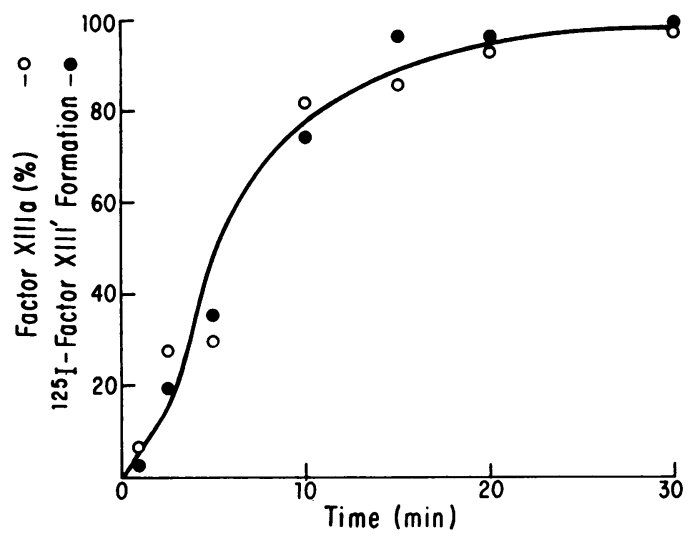

Figure 3. Time course of thrombin cleavage and activation of ${ }^{125} \mathrm{I}-$ Factor XIII and native plasma Factor XIII. ${ }^{125} \mathrm{I}$-Factor XIII $(0.5 \mu \mathrm{g}$, $3,620 \mathrm{cpm} / \mathrm{ng}$ ) was added to PPP diluted to a final concentration of $20 \%$ (vol/vol). Gly-Pro-Arg-Pro ( $2 \mathrm{mM}$ ) was added, followed by thrombin $(0.50 \mathrm{U} / \mathrm{ml})$ and calcium chloride $(5 \mathrm{mM})$. At intervals, 0.050-ml aliquots were removed and subected to SDS-10\% PAGE under nonreducing conditions or assayed for Factor XIIIa after inactivation of thrombin with heparin and PNPGB. The radioactivity in gel slices corresponding to the a- and $\mathrm{a}^{\prime}$-chains was measured, and the percentage of Factor XIII' formed was calculated as described in Fig. 2. Factor XIIIa was assayed by the monodansylcadaverine assay. Each time point was assayed in duplicate. The percentage of the total functional Factor XIII in the plasma was determined by dividing the value obtained at each time point by the value obtained from maximally activating the plasma with $20 \mathrm{U} / \mathrm{ml}$ thrombin for $15 \mathrm{~min} .{ }^{125} \mathrm{I}$ Factor XIII represented $15 \%$ of the total Factor XIII activity in the plasma sample.

activity in thrombin-treated plasma correlated with the amount of cleavage of ${ }^{125} \mathrm{I}$-Factor XIII added to the plasma. The results of these experiments demonstrate that ${ }^{125}$ I-Factor XIII was a suitable marker for native plasma Factor XIII.

Time course of thrombin cleavage of Factor XIII and fibrinogen during in vitro clotting. We determined the time course of thrombin cleavage of fibrinogen and Factor XIII in PRP in the absence of anticoagulant. As shown in Fig. 4, a visible clot formed after $75 \mathrm{~min}$ of incubation. Subsequent to clotting, thrombin cleavage of FPA from fibrinogen and cleavage of the a-chain of Factor XIII paralleled each other (Fig. 4). These results suggest that thrombin cleavage of Factor XIII is closely correlated with cleavage of fibrinogen by thrombin during in vitro clotting of PRP.

When the rate of thrombin cleavage of fibrinogen and Factor XIII are compared by measuring the slope of the curves in Fig. 4, fibrinogen is cleaved more rapidly $(226 \mathrm{pmol} / \mathrm{min}$ per $\mathrm{ml}$ ) than the a-chains of Factor XIII ( $5.3 \mathrm{pmol} / \mathrm{min}$ per $\mathrm{ml})$. Neither the $K_{\mathrm{m}}$ or $V_{\max }$ of these reactions has been quantitated in this study in that the thrombin concentration in clotting plasma changes continuously throughout the incubation.

${ }^{125}$ I-Factor XIIIa binding to fibrin. When ${ }^{125}$ I-plasma Factor XIII was added to $0.9 \mathrm{ml}$ of citrated PPP and then recalcified, we found that the a-chains were absent from the serum while the b-chains remained in the serum (Fig. 5, lane $C$ ).

To determine the nature of the material bound to the clot, it was solubilized in SDS sample buffer under nonreducing conditions and subjected to SDS-PAGE (Fig. 5, lane B). All of the radioactivity bound to fibrin migrated as a broad band

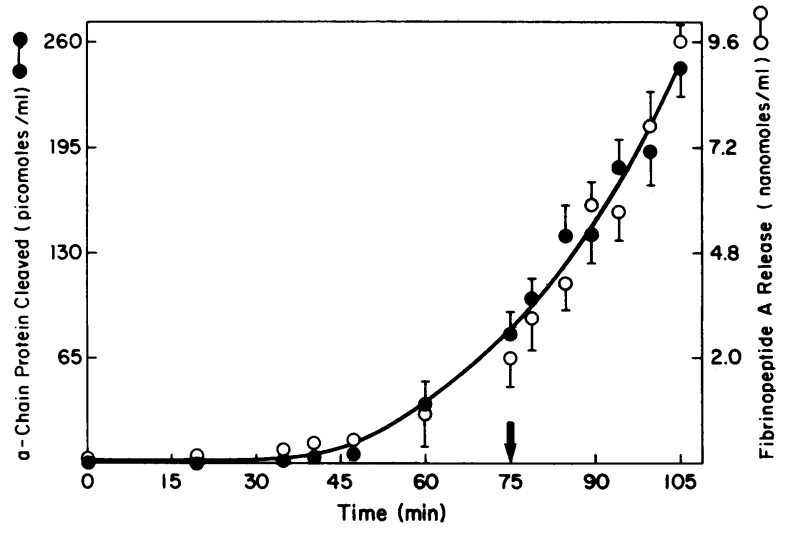

Figure 4. Time course of thrombin cleavage of Factor XIII and fibrinogen in PRP. Factor XIII $(1 \mu \mathrm{g} ; 6,150 \mathrm{cpm} / \mathrm{ng})$ was added to $1.0-\mathrm{ml}$ aliquots of PRP and allowed to clot at $22^{\circ} \mathrm{C}$ in polystyrene tubes. After adding inhibitors, samples were assayed at various times for Factor XIII' formation ( $\bullet$ ). In a parallel experiment, samples of PRP were assayed for FPA release (o). The average and standard deviation for each time point were calculated. The nanomoles of FPA released and the picomoles of Factor XIII a-chain cleaved by thrombin were calculated as described in Methods. The arrow indicates the time when the entire sample clotted. At $60 \mathrm{~min}$, visible fibrin strands were observed but the entire sample did not gel until $75 \mathrm{~min}$. The concentration of fibrinogen and Factor XIII a-chain in the incubation mixture were $7 \times 10^{-6} \mathrm{M}$ and $2.6 \times 10^{-7} \mathrm{M}$, respectively.

in the $\mathrm{a}^{\prime}$-chain position of the gel in front of the a-chains $\left(M_{\mathrm{r}}\right.$ $=72,000-78,000)$. The broadness of the band varied depending upon the amount of protein added to the gel and was not further investigated.

To demonstrate optimal conditions for washing unbound ${ }^{125} \mathrm{I}$-Factor XIII from the clots, citrated plasma containing 1 $\mu \mathrm{g} / \mathrm{ml}$ of ${ }^{125} \mathrm{I}$-plasma Factor XIII $(2,175 \mathrm{cpm} / \mathrm{ng})$ was recalcified. The clots were compressed to remove excess fluid. There were $6.0 \times 10^{5} \mathrm{cpm}$ in the clot and $1.525 \times 10^{6} \mathrm{cpm}$ in the serum. The serum contained mostly b-chains, and $<8 \%$ of the $a^{\prime}-$ chains. The clot was then placed in $2 \mathrm{ml}$ of fresh Tris-buffered saline every $5 \mathrm{~min}$. The first through fourth washes, which contained $<10 \%$ of the radioactivity initially present in the clot, were composed entirely of b-chains. Thus thrombincleaved a-chains are bound exclusively to fibrin and were not lost during washing. We cannot rule out the possibility that the $a_{2}^{\prime} b_{2}$ intermediate is bound initially to fibrin and that binding itself causes rapid dissociation of the $b_{2}$-subunit, and release into the serum. When $1 \mu \mathrm{g}$ of ${ }^{125} \mathrm{I}$-human serum albumin was added to citrated plasma, which was then clotted by recalcifying the sample or adding thrombin, $0.8 \%$ was bound to the clot. Therefore, binding of ${ }^{125} \mathrm{I}$-Factor XIII' or ${ }^{125}$ I-Factor XIIIa, was not due to nonspecific trapping.

To demonstrate that the thrombin-cleaved a-chains could be completely eluted from the clot, $1 \mathrm{ml}$ of citrated plasma containing $1 \mu \mathrm{g}$ of ${ }^{125} \mathrm{I}$-plasma Factor XIII was recalcified and the clots that formed were dissolved in SDS sample buffer and incubated for various intervals at $65^{\circ} \mathrm{C}$. The percentage of the initial radioactivity eluted was then determined. $98 \%$ of the bound ${ }^{125}$ I-Factor XIIIa was eluted from the clot after $18 \mathrm{~h}$ of incubation. In addition, no high molecular complexes of ${ }^{125} \mathrm{I}$ Factor XIII were detected by SDS-PAGE.

To obtain additional evidence that the ${ }^{125}$ I-Factor XIII was 


\section{Origin}

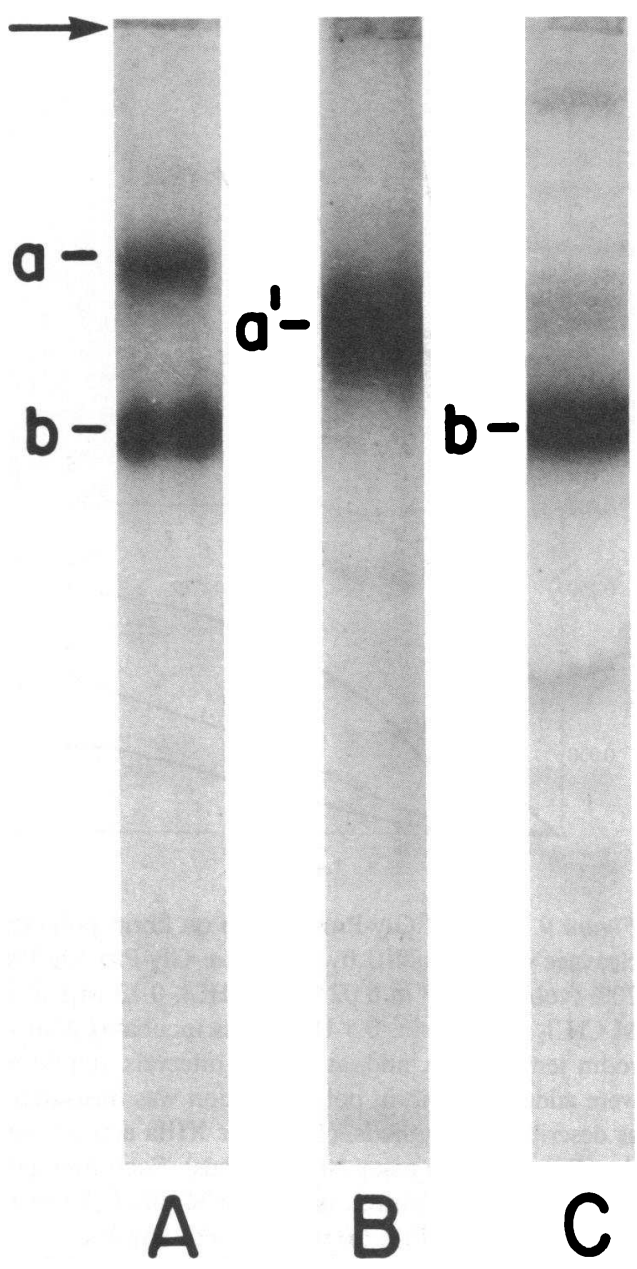

Figure 5. Binding of Factor XIIIa to fibrin. ${ }^{125}$ I-plasma Factor XIII ( $1 \mu \mathrm{g} ; 4,985 \mathrm{cpm} / \mathrm{ng}$ ) was added to $0.9 \mathrm{ml}$ of citrate-anticoagulated PPP which was then recalcified with $0.1 \mathrm{ml}$ of $100 \mathrm{mM}$ calcium chloride. Once the clot formed, it was compressed and placed in 0.5 $\mathrm{ml}$ of clot-solubilizing buffer containing SDS and urea (described in Methods) for $16 \mathrm{~h}$ at $56^{\circ} \mathrm{C}$. Then, $25 \mu \mathrm{l}$ of the solubilized clot was electrophoresed in a $10 \%$ SDS-PAGE gel. The serum was mixed with an equal volume of the sample buffer and electrophoresed similarly. Lane $A$ shows the autoradiogram of ${ }^{125}$ I-Factor XIII in plasma. Lane $B$ is the autoradiogram of the washed and solubilized clot. Lane $C$ is the autoradiogram of the serum sample. Each sample contained $\sim 40,000 \mathrm{cpm}$. The autoradiograms were developed for $16 \mathrm{~h}$.

an accurate marker for the unlabeled Factor XIII in plasma, we measured the time course of disappearance of Factor XIIIa activity from serum, and the binding of ${ }^{125} \mathrm{I}$-Factor XIIIa to the fibrin clot (Fig. 6). The molar ratio of ${ }^{125}$ I-Factor XIII to unlabeled Factor XIII was $1: 15$ in these experiments. Before the clot was visible at $60 \mathrm{~min}, 8 \%$ of the ${ }^{125} \mathrm{I}$-Factor XIII was converted to ${ }^{125} \mathrm{I}$-Factor XIII' in plasma and $100 \%$ of the original Factor XIII activity remained in the plasma. At several time points after the clot formed, there was parallel disappearance of Factor XIII activity associated with binding of thrombincleaved ${ }^{125} \mathrm{I}$-Factor XIII a-chains to the fibrin clot (Fig. 5). These data indicate that ${ }^{125} \mathrm{I}$-labeled and unlabeled Factor XIII behave similarly during clotting. In addition, either Factor XIIIa becomes rapidly associated with the fibrin clot during in vitro clotting or Factor XIII bound to fibrin is converted to Factor XIIIa.

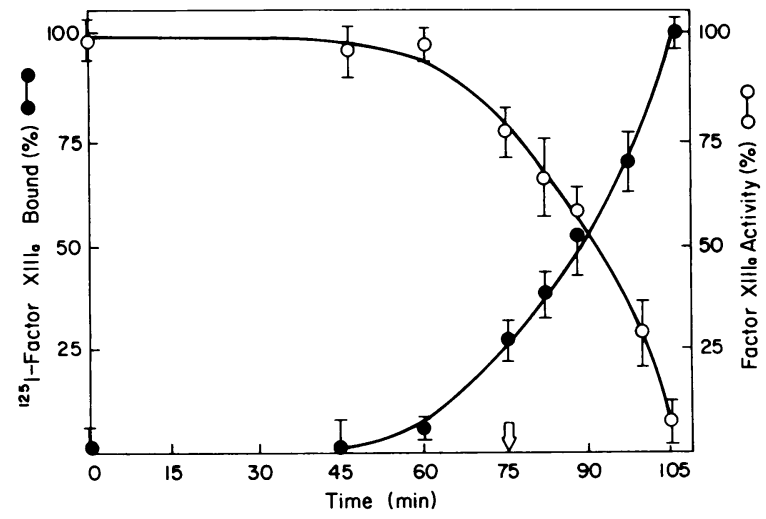

Figure 6. Binding of ${ }^{125} \mathrm{I}$-Factor XIIIa to the fibrin clot and measurement of Factor XIIIa in serum. The time course of binding of ${ }^{125} \mathrm{I}$ Factor XIIIa to fibrin and disappearance of Factor XIIIa activity from serum were determined during in vitro clotting. ${ }^{125} \mathrm{I}$-plasma Factor XIII $(1 \mu \mathrm{g} ; 5,320 \mathrm{cpm} / \mathrm{ng})$ was added to $1.0 \mathrm{ml}$ of PRP and allowed to clot spontaneously in polystyrene tubes at $22^{\circ} \mathrm{C}$. Fibrin strands were first detected at $60 \mathrm{~min}$. The samples did not clot completely until 75 min (arrow). Plasma and serum samples were assayed for Factor XIII activity (O) at various intervals as described in Methods. Binding of ${ }^{125} \mathrm{I}$-Factor XIIIa to fibrin was determined by counting the amount of radioactivity in the washed clot $(\bullet)$. At all time points $>95 \%$ of the radioactivity in the clot migrated in the $a^{\prime}$-chain area. Therefore, the total amount of radioactivity bound was assumed to represent Factor XIIIa.

Time course of cleavage of Factor XIII and fibrinogen in PPP containing Gly-Pro-Arg-Pro. When thrombin $(1 \mathrm{U} / \mathrm{ml})$ was incubated with $20 \%$ plasma in $0.02 \mathrm{M}$ Tris- $\mathrm{HCl}, 0.13 \mathrm{M}$ $\mathrm{NaCl}, \mathrm{pH} \mathrm{7.4,} 2 \mathrm{mM}$ Gly-Pro-Arg-Pro completely inhibited fibrin monomer polymerization for $15 \mathrm{~min}$. The absence of clotting allowed simultaneous measurement of Factor XIIIa activity and fibrin formation. Gly-Pro-Arg-Pro at concentrations between 0.1 and $2.0 \mathrm{mM}$ did not modify Factor XIIIa activity or the rate of release of FPA from fibrinogen by thrombin in PPP. When fibrin polymerization was inhibited by Gly-ProArg-Pro, thrombin cleavage of fibrinogen occurred before cleavage of Factor XIII (Fig. 7).

Because cleavage of fibrinogen was shown to occur before cleavage of Factor XIII, we investigated whether the thrombin concentrations required to cleave fibrinogen and Factor XIII were different. When thrombin was added to PPP containing Gly-Pro-Arg-Pro, more fibrinogen was cleaved than Factor XIII (Fig. 8). One-half of the plasma fibrinogen and Factor XIII were cleaved at $15 \mathrm{~min}$ at $0.35 \mathrm{U} / \mathrm{ml}$ and $0.65 \mathrm{U} / \mathrm{ml}$ of thrombin, respectively, in the presence of Gly-Pro-Arg-Pro.

The effect of various concentrations of Gly-Pro-Arg-Pro were also investigated. The rate of thrombin-catalyzed Factor XIIIa formation was progressively inhibited by increasing concentrations of Gly-Pro-Arg-Pro (Fig. 9 B). In contrast, fibrinogen was cleaved at a similar rate at each Gly-Pro-ArgPro concentration. The percentage of thrombin-catalyzed FPA release was $38 \% \pm 12(\mathrm{SD})$ at $15 \mathrm{~s}, 68 \% \pm 18$ at $30 \mathrm{~s}$, and $92 \% \pm 12$ at $60 \mathrm{~s}$ at each concentration of Gly-Pro-Arg-Pro.

\section{Discussion}

In this study we compared the time course of thrombin cleavage of Factor XIII and fibrinogen during in vitro clotting. 


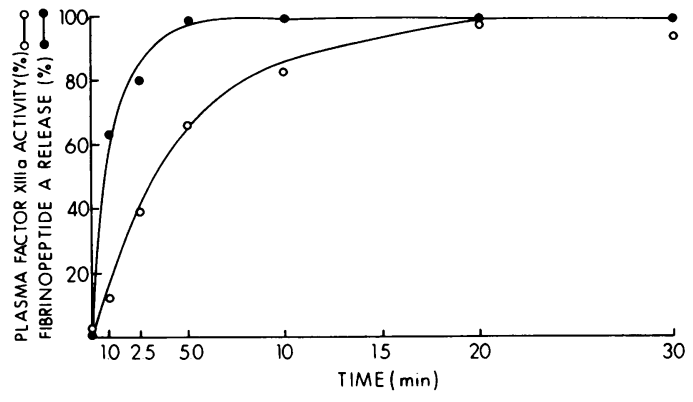

Figure 7. Time course of cleavage of Factor XIII and fibrinogen by thrombin in PPP containing Gly-Pro-Arg-Pro. Thrombin $(1.0 \mathrm{U} / \mathrm{ml})$ was incubated with $20 \%$ (vol/vol) plasma in $0.02 \mathrm{M}$ Tris- $\mathrm{HCl}, 0.13$ $\mathrm{M} \mathrm{NaCl}, \mathrm{pH} \mathrm{7.7}$, containing $2 \mathrm{mM}$ Gly-Pro-Arg-Pro. At various intervals, aliquots were removed, inhibitors of thrombin added (heparin, $1.0 \mathrm{U} / \mathrm{ml}$ and PNPGB, $0.1 \mathrm{mM}$ ) and the samples assayed for FPA and Factor XIIIa. Factor XIIIa activity (0) and FPA release (•) are expressed as the percentage of the amount found in plasma incubated with $20 \mathrm{U} / \mathrm{ml}$ of thrombin for $15 \mathrm{~min}$ in otherwise identical conditions. Each time point was assayed in duplicate; the results represent the average. This is one of five such experiments.

Using ${ }^{125}$ I-plasma Factor XIII of high specific radioactivity, we followed cleavage of the Factor XIII by thrombin during spontaneous clotting of PRP. The cleavage peptide was not radiolabeled under these conditions, and its release therefore cannot be used to assay its cleavage by thrombin. Native radiolabeled a-chains and thrombin-cleaved a-chains were separated by electrophoresis in $10 \%$ SDS-PAGE gels. Since a- and b-chains migrate identically under reducing conditions in the discontinuous SDS buffer system used for this study, the proteins were electrophoresed under nonreducing conditions. We were able to separate reproducibly the a- and a'-chains by this technique. There were two limitations to this method. First, $\sim 12 \%$ of the radioactivity always migrated in the original a-chain position even after complete activation of the plasma Factor XIII. The radioactivity migrating in the original achain region probably represents trailing radioactivity although

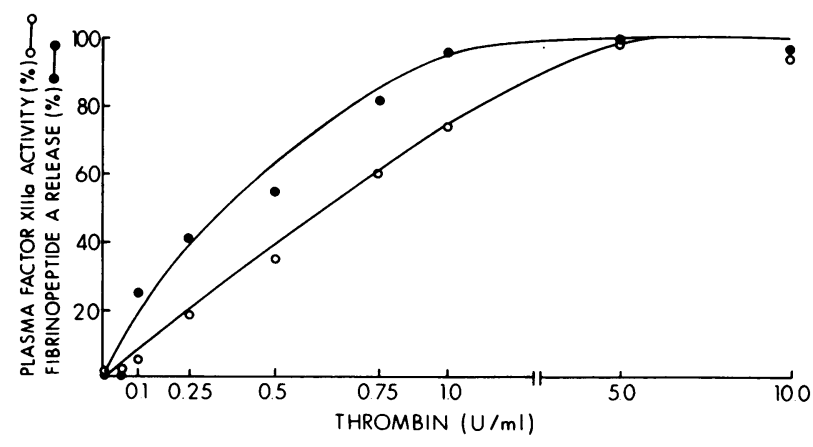

Figure 8. Effect of thrombin on cleavage of Factor XIII and fibrinogen in PPP containing Gly-Pro-Arg-Pro. Thrombin (0.05 to 10.0 $\mathrm{U} / \mathrm{ml}$ ) was incubated with $20 \%$ (vol/vol) plasma in $0.02 \mathrm{M}$ Tris- $\mathrm{HCl}$, $0.13 \mathrm{M} \mathrm{NaCl}, \mathrm{pH} 7.4$, containing $1 \mathrm{mM}$ Gly-Pro-Arg-Pro for $15 \mathrm{~min}$ at $37^{\circ} \mathrm{C}$. The reaction was stopped as described in Fig. 6, and aliquots assayed for FPA release (•) and Factor XIIIa activity (o). Results are expressed as the percentage of the amount found in plasma incubated with $20 \mathrm{U} / \mathrm{ml}$ of thrombin for $15 \mathrm{~min}$. Each time point was assayed in duplicate. The results are representative of three such experiments.
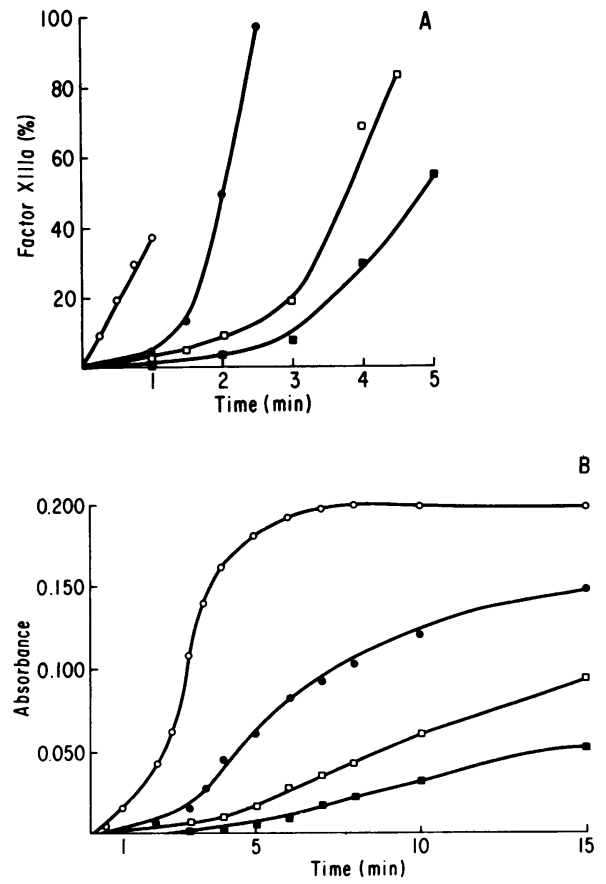

Figure 9. Effect of Gly-Pro-Arg-Pro on fibrin polymerization and cleavage of Factor XIII by thrombin. Gly-Pro-Arg-Pro was added to $20 \%$ (vol/vol) PPP in $0.02 \mathrm{M}$ Tris- $\mathrm{HCl}, 0.13 \mathrm{M} \mathrm{NaCl}, \mathrm{pH} 7.4,0.005$ $\mathrm{M} \mathrm{CaCl}_{2}$. Thrombin, $0.5 \mathrm{U} / \mathrm{ml}$, was incubated with the plasma at room temperature, and, at various intervals, inhibitors of thrombin were added. $(A)$ Fibrin polymerization was measured at OD $650 \mathrm{~nm}$ as described in Methods. (B) Factor XIIIa activity was measured by the $\left[{ }^{3} \mathrm{H}\right]$ putrescine assay (see Methods). Each time point was assayed in duplicate. (০) Control. (॰) $0.25 \mathrm{mM}$ Gly-Pro-Arg-Pro. (口) 0.50 mM Gly-Pro-Arg-Pro. (口) 0.75 Gly-Pro-Arg-Pro.

it is possible that $12 \%$ of the ${ }^{125} \mathrm{I}$-a-chains is insensitive to thrombin.

The second limitation of the technique is that simultaneous measurements of FPA and Factor XIIIa formation could not be made due to the radioactivity in the ${ }^{125}$ I-Factor XIII that prevented FPA measurement by radioimmunoassay. Since simultaneous measurements could not be made, small differences in the time course of cleavage of fibrinogen and Factor XIII by thrombin may have been undetected. A major advantage of using ${ }^{125}$ I-Factor XIII to follow cleavage by thrombin in PRP is that measurements could be made during the course of spontaneous clotting.

Our findings that $(a)$ the time course of thrombin cleavage of ${ }^{125}$ I-Factor XIII and the generation of Factor XIIIa activity was similar, and $(b)$ the disappearance of native Factor XIII from clotting plasma, correlated with binding of ${ }^{125} \mathrm{I}$-Factor XIIIa to fibrin, indicate that ${ }^{125}$ I-Factor XIII behaved similarly to native Factor XIII. Moreover, the time course of thrombin cleavage of ${ }^{125} \mathrm{I}$-Factor XIII and native Factor XIII was similar.

Because the fibrin clot selectively bound the thrombincleaved a-chains of ${ }^{125} \mathrm{I}$-Factor XIII', we were able to quantitate their formation by washing clots free of unbound protein, solubilizing the clots, and quantifying the amount of radioactivity in the region of the $a^{\prime}$-chain in 10\% SDS-PAGE gels.

We took advantage of the slow clotting that occurs in polystyrene test tubes to follow the time course of thrombin cleavage of Factor XIII and fibrinogen. There was a similar 
percentage of the total fibrinogen and Factor XIII cleaved by thrombin when measured at several time points after clotting occurred in PRP.

We did not measure the $K_{\mathrm{m}}$ or $V_{\max }$ for the individual reactions in this study. We were primarily interested in the time course of thrombin cleavage of Factor XIII and fibrinogen in a physiologic plasma system. When the data in Fig. 4 are expressed as moles of Factor XIII a-chain or fibrinogen cleaved/ minute per milliliter, fibrinogen is cleaved by thrombin at a 43-fold higher rate than Factor XIII. Inasmuch as fibrinogen was present in a 27 -fold molar excess compared to Factor XIII a-chains, the differences in the rate may represent a difference in the initial substrate concentrations.

Thrombin cleavage of fibrinogen preceded cleavage of Factor XIII when Gly-Pro-Arg-Pro was used to inhibit fibrin polymerization. Gly-Pro-Arg-Pro delayed activation of Factor XIII in a concentration-dependent manner. Cleavage of fibrinogen was not affected at identical concentrations of Gly-ProArg-Pro. These results probably account for the differences observed in the relative time course of activation of Factor XIII and fibrinogen between spontaneously clotting PRP and PPP containing Gly-Pro-Arg-Pro. In any event, the results obtained in spontaneously clotting plasma more closely resemble the physiologic situation.

The effect of platelets on activation of Factor XIII during clotting has not been determined. Thus the presence of platelets may be responsible for some of the differences observed in the two types of experiments. Other differences in the two experimental systems that may have affected the results include the concentrations of calcium and thrombin and diluted versus undiluted plasma.

Janus et al. (8) have studied the time course of cleavage of fibrinogen and Factor XIII by thrombin using high-pressure liquid chromatography to measure fibrinopeptide $\mathrm{A}$ and the activation peptide from Factor XIII. These investigators found that Factor XIII was cleaved by thrombin after cleavage of fibrinogen in dilute solutions of these substrates containing polyethylene glycol. Our results using PPP and Gly-Pro-ArgPro are very similar to theirs.

There are several possible mechanisms by which Gly-ProArg-Pro inhibits formation of Factor XIIIa. These include: $(a)$ inhibition of the assembly of fibrin monomers, $(b)$ inhibition of thrombin binding to the Factor XIII-fibrinogen (fibrin) complex, and $(c)$ inhibition of Factor XIII binding to fibrinogen. In preliminary experiments, we have found that Factor XIIIa formation is delayed when fibrin polymerization is inhibited by glycerol or 1,6-hexanediol. Furthermore, when fibrinogen is modified by acetylation or treatment with methylene blue and irradiation, polymerization and Factor XIIIa formation are both inhibited. These results suggest that the effect of GlyPro-Arg-Pro on Factor XIIIa formation is not unique to the peptide and that other modifications that inhibit fibrin polymerization also modify cleavage of Factor XIII by thrombin.

Recent evidence indicates that at low thrombin concentrations, the $\mathrm{A} \alpha$ and $\mathrm{B} \beta$ chains of fibrinogen behave independently in their competition for thrombin (22). These results are in contrast to those of Hurlet-Jensen et al. (23), who found that when fibrin polymerization occurs at higher thrombin concentrations, the initial rate of release of FPA is faster than FPB. These investigators have also shown that fibrin polymerization enhances release of FPB by thrombin (23). Fibrin polymerization may have a similar effect on thrombin-catalyzed Factor
XIIIa formation. In that we did not measure FPB release in our experiments, we do not know whether it was cleaved before or after cleavage of Factor XIII by thrombin. However, others have reported that FPB release occurs after cleavage of Factor XIII by thrombin (8). In addition, in preliminary studies, we have found that purified fibrin monomers devoid of FPA and FPB facilitate thrombin-catalyzed formation of Factor XIIIa (24). Moreover, Gly-Pro-Arg-Pro inhibited enhancement of Factor XIIIa formation by the purified fibrin monomers. Thus, FPB release does not appear to play an important role in regulating Factor XIIIa formation. Additional studies using purified Factor XIII and fibrinogen are in progress to define further the effects of the thrombin and Gly-Pro-ArgPro concentrations on the kinetics of FPA and FPB release, and cleavage of Factor XIII.

Our results with plasma, a complex biological fluid, indicate that cleavage of Factor XIII by thrombin is closely coordinated with cleavage of FPA from fibrinogen, and that conversion of fibrinogen to polymerized fibrin may facilitate activation of Factor XIII.

\section{Acknowledgments}

We thank Mr. James Harris for his assistance in the preparation of this manuscript, and acknowledge the expert technical assistance of Mrs. Eileen Matthews, Mr. Michael Safrin, and Mr. Joseph V. Dobson. We appreciate the helpful suggestions of Dr. Pat McKee and Dr. Salvatore Pizzo.

This research work was supported in part by grants HL-21403, CA-22202, HL-32342, and T32 HL-07100 from the National Institutes of Health, grants-in-aid from the American Heart Association and its Connecticut (83-957) and North Carolina Affiliates, and the Medical Research Service of the Veterans Administration. Dr. Shuman is a recipient of Research Career Development Award 1K04 HL-00802 from the National Institutes of Health. Dr. Greenberg was an American Heart Association Research Fellow, Northern California Affiliate, during the course of this research.

\section{References}

1. Blombäck, B., and A. Vestermark. 1958. Isolation of fibrinopeptides by chromatography. Ark. Kemi. 12:173-182.

2. Blombäck, B., B. Hessel, D. Hogg, and L. Lisbeth Therkildsen. 1978. A two-step fibrinogen-fibrin transition in blood coagulation. Nature (Lond.). 275:501-505.

3. Hermans, J., and J. McDonagh. 1982. Fibrin: Structure and interactions. Semin. Thromb. Hemostasis. 8:11-24.

4. Lorand, L., and R. B. Credo. Thrombin and fibrin stabilization. 1977. In Chemistry and Biology of Thrombin. R. Lundbald, J. W. Fenton, and K. G. Mann, editors. Ann Arbor Science, Ann Arbor, MI. 311-323.

5. Takagi, T., and R. F. Doolittle. 1974. Amino acid sequence studies on Factor XIII and peptide released during its activation by thrombin. Biochemistry. 13:750-756.

6. Curtis, C. G., K. L. Brown, R. B. Credo, R. A. Domanik, A. Gray, P. Stenberg, and L. Lorand. 1974. Calcium-dependent unmasking of active center cysteine during activated fibrin stabilizing factor. Biochemistry. 13:3774-3780.

7. Credo, R. B., C. G. Curtis, and L. Lorand. 1978. $\mathrm{Ca}^{2+}$-related regulatory function of fibrinogen. Proc. Natl. Acad. Sci. USA. 75: 4234-4237.

8. Janus, T. J., S. D. Lewis, L. Lorand, and J. A. Shafer. 1983. Promotion of thrombin-catalyzed activation of Factor XIII by fibrinogen. Biochemistry. 26:6269-6272. 
9. Dvilansky, A., A. F. H. Britten, and A. G. Loewy. 1970. Factor XIII assay by an isotope method. Br. J. Haematol. 18:399-404.

10. Ikematsu, S., R. P. McDonagh, H. M. Reisner, C. Skrzynia, and J. McDonagh. 1981. Immunochemical studies of human Factor XIII-radioimmunoassay of the carrier subunit of the zymogen. J. Lab. Clin. Med. 97:662-676.

11. Skrzynia, C., H. M. Reisner, and J. McDonagh. 1982. Characterization of the catalytic subunit of Factor XIII by radioimmunoassay. Blood. 60:1089-1095.

12. Folk, J. E., and J. S. Finlayson. 1977. The $\epsilon$ (glutamyl)lysine cross-link and the catalytic role of transglutaminase. Adv. Protein Chem. 31:1-133.

13. Curtis, C. G., and L. Lorand. 1976. Fibrin-stabilizing factor (Factor XIII). Methods Enzymol. 45:177-191.

14. Schwartz, M. L., S. V. Pizzo, R. L. Hill, and P. A. McKee. 1973. Human Factor XIII from plasma and platelets. Molecular weights, subunit structures, proteolytic activation, and cross-linking of fibrinogen and fibrin. J. Biol. Chem. 248:1395-1407.

15. Greenberg, C. S., and M. A. Shuman. 1982. The zymogen forms of blood coagulation Factor XIII bind specifically to fibrinogen. J. Biol. Chem. 257:6096-6101.

16. Mihayil, E. 1968. Physicochemical studies of bovine fibrinogen. Ultraviolet absorption and its relation to the structure of the molecule. Biochemistry. 7:208-223.

17. Laemmli, U. K. 1970. Cleavage of structural proteins during the assembly of the head of bacteriophage T4. Nature (Lond.). 227: 680-685.

18. Lorand, L., T. Urayama, J. W. C. de Kiewiet, and H. L. Nossel. 1969. Diagnostic and genetic studies on fibrin stabilizing factor with a new assay based on amine incorporation. J. Clin. Invest. 48: 1054-1064.

19. Lorand, L., L. K. Campbell-Wilkes, and L. Cooperstein. 1972. A filter paper assay for transamidating enzymes using radioactive amine substrates. Anal. Biochem. 50:623-631.

20. Myers, T. J., F. R. Rickles, C. Barb, and M. Cronlund. 1981. Fibrinopeptide $\mathrm{A}$ in acute leukemia: Relationship of activation of blood coagulation to disease activity. Blood. 57:518-524.

21. Miraglia, C. C., and C. S. Greenberg. 1985. Measurement of blood coagulation Factor XIIla formation in plasma containing glycylL-prolyl-L-arginyl-L-proline. Anal. Biochem. 144:165-171.

22. Hanna, L. S., H. A. Scheiaga, C. W. Francis, and V. J. Marder. 1984. Comparison of structures of various human fibrinogens and a derivative thereof by a study of the kinetics of release of fibrinopeptides. Biochemistry. 23:4681-4687.

23. Hurlet-Jensen, A., H. Z. Cummins, H. L. Nossel, and C. Y. Liu. 1982. Fibrin polymerization and release of fibrinopeptide B by thrombin. Thromb. Res. 27:419-427.

24. Greenberg, C. S., C. C. Miraglia, J. V. Dobson, and K. E. Achyuthan. 1984. Fibrin polymerization regulates thrombin cleavage of plasma Factor XIII. Blood. 64:263a. (Abst.) 